Itinéraires Itinéraires

Littérature, textes, cultures

2012-2 | 2012

Intime et politique

\title{
La révolution palestinienne à l'épreuve de l'intime : Un captif amoureux de Jean Genet
}

\author{
Myriam Bendhif-Syllas
}

\section{(2) OpenEdition}

1 Journals

Édition électronique

URL : http://journals.openedition.org/itineraires/1150

DOI : $10.4000 /$ itineraires. 1150

ISSN : 2427-920X

Éditeur

Pléiade

\section{Édition imprimée}

Date de publication : 1 novembre 2012

Pagination : 119-130

ISBN : 978-2-336-00027-5

ISSN : $2100-1340$

\section{Référence électronique}

Myriam Bendhif-Syllas, «La révolution palestinienne à l'épreuve de l'intime: Un captif amoureux de Jean Genet », Itinéraires [En ligne], 2012-2 | 2012, mis en ligne le 01 novembre 2012, consulté le 29 septembre 2020. URL : http://journals.openedition.org/itineraires/1150 ; DOI : https://doi.org/ 10.4000/itineraires. 1150

\section{(c) (i) $(9$}

Itinéraires est mis à disposition selon les termes de la licence Creative Commons Attribution - Pas d'Utilisation Commerciale - Pas de Modification 4.0 International. 


\title{
La révolution palestinienne à l'épreuve de l'intime : Un captif amoureux de Jean Genet
}

\begin{abstract}
Two long stays in the Palestinian camps will allow the writer Jean Genet to be one of the rare Westerners to be able to testify of the life of the insurgents. This testimony, at the same time essay and autobiography, is entitled A Loving Captive. Far from the expected panegyric, from the committed paper waited by the defenders of the Palestinian cause, Genet distances himself: he will not be a soldier of the revolution, he will not be one of the thinkers. Indeed, he will define himself rather as an observer and a storyteller while giving his own political vision of the conflict. It is at the heart of the intimate that Genet intends to find his vision of Palestine: he articulates the memory with the fiction in a "report-mirror." Defender of a revolution while keeping a critical and outside position, outside the history while living it, Jean Genet reports an intimate experience, that of a man and an artist in a people with whom he desperately fell in love.
\end{abstract}

Keywords : Genet, intimate, Palestinian revolution, Loving Captive, memory, politics Mots clés : Genet, intime, révolution palestinienne, Un captif amoureux, souvenir, politique

Deux longs séjours dans les camps palestiniens permettront à l'écrivain Jean Genet d'être l'un des rares Occidentaux à pouvoir témoigner de la vie des insurgés. Ce document inclassable, à la fois essai et autobiographie, s'intitule Un captif amoureux ${ }^{1}$. Genet y entremêle de façon tout à fait inédite des notations historiques précises, des analyses politiques, des dialogues reconstitués, des anecdotes et des souvenirs de différentes périodes de sa vie. Or, Genet fait tout pour convaincre de l'authenticité de son projet initial, des Mémoires d'un genre nouveau. En effet, il intitule respectivement les deux parties de son œuvre « Souvenirs I " et « Souvenirs II ». Or, le souvenir, la mémoire, comme l'événement historique, la vision politique, ne seront pas à prendre au pied de la lettre, mais bel et

1. Jean Genet, Un captif amoureux, Paris, Gallimard, coll. « Folio », 1986. 
bien à passer au crible de la fiction qui les recompose. L'objet de cette étude sera de montrer l'articulation entre les notions d'intime et de politique dans cette œuvre posthume; articulation qui met au jour un jeu de cache-cache entre Genet et son lecteur. Dans ce jeu, l'auteur multiplie les déclarations contradictoires et fausse peu à peu le projet affiché à l'origine. Au cœur de la politique s'affirme l'intime des hommes, qui est peutêtre son seul reflet possible.

\section{La fiction d'une œuvre engagée}

Genet revient à plusieurs reprises sur l'origine du Captif amoureux. L'enjeu en est clairement politique puisqu'il s'agissait, pour l'écrivain, d'apporter un témoignage sur le combat livré par les Palestiniens contre Israël, mais aussi contre le pouvoir jordanien. Le Captif devait être un ouvrage engagé, une arme de plus dans cette lutte guerrière. Le livre serait tout d'abord issu d'une commande faite par Yasser Arafat lors du premier séjour de Genet au Moyen-Orient en 1970². Projet auquel l'écrivain ne donnera pas suite mais qui lui permettra d'obtenir du leader palestinien un laissez-passer lui octroyant une totale liberté de circulation sur les territoires de ''OLP $^{3}$. Ensuite, des amis palestiniens ${ }^{4}$ enjoignent Genet d'écrire sur leur combat, en particulier pour réagir aux massacres perpétrés à Sabra et Chatila ${ }^{5}$ en 1982 et dont Genet fut témoin.

Genet se démarque du panégyrique espéré par Yasser Arafat, mais aussi de l'écrit engagé attendu par les défenseurs de la cause palestinienne : il ne sera pas un soldat de la révolution, il n'en sera pas même l'un des penseurs, ni l'un de ses propagandistes. En effet, s'il soutient pleinement cette cause, il se définit avant tout comme un observateur et un conteur. «Genet refuse d'avoir la tete politique, même si ce sont des groupes politiques qu'il aime et qu'il observe ${ }^{6}$. » Genet reste en dehors du conflit qu'il aborde de l'intérieur, certes, mais il demeure irrémédiablement dans ses marges : « ma non-appartenance à une nation, à une action où je ne me confondis jamais ${ }^{7} »$.

2. Ibid., p. 37 et p. 150 .

3. Edmund White, Jean Genet, Paris, Gallimard, 1993, p. 546.

4. Entretien avec Leïla Shahid, dans Jérôme Hankins, Genet à Chatila, Paris, Solin, 1992 , p. 24. Voir également Jean Genet, Un captif amoureux, op. cit., p. 400-401.

5. Genet rédigera un essai percutant sur ces massacres : «Quatre heures à Chatila », repris dans L'Ennemi déclaré : textes et entretiens, éd. Albert Dichy, Paris, Gallimard, 1991, p. 243-267.

6. Annie Cohen-Solal, «Jean sans Terre. Qui s'étonnera que Jean Genet nous ait laissé une bombe en guise de testament? ", Le Nouvel Observateur, 23-29 mai 1986, repris dans Agnès Fontvieille-Cordani et Dominique Carlat (dir.), Jean Genet et son lecteur, Autour de la réception critique de Journal du voleur et Un captif amoureux, Saint-Étienne, Publications de l'Université de Saint-Étienne, 2010, p. 114.

7. Jean Genet, Un captif amoureux, op. cit., p. 150. 
De ce fait, Un captif amoureux remet complètement en question la notion d'engagement littéraire ${ }^{8}$. S'il se positionne en ami des Palestiniens, Genet n'entend pas jouer le rôle d'un Sartre du désert. Il plaide la cause palestinienne jusqu'à une certaine limite.

Si ma décision de rendre publiques mes années avec la résistance m'indiquait qu'elle s'éloigne? C'est qu'un innommable sentiment m'avertit : la révolte s'estompe, elle se lasse, va tourner dans le sentier et disparaître. On fera d'elle des chansons héroïques. C'est que j'ai regardé la résistance comme si elle allait disparaître demain ${ }^{9}$.

Comme l'Algérie, comme d'autres pays, oubliant la révolution dans le monde arabe, elle ne songeait qu'au territoire sur lequel un vingt-deuxième État naîtrait, apportant avec lui ce qu'on exige d'un nouveau venu : l'Ordre, la Loi. Cette révolte si longtemps hors-la-loi, aspirait-elle à devenir loi dont le Ciel serait l'Europe ${ }^{10}$ ?

En elle, il admire la révolte et le tragique d'un combat qui semble voué à l'échec. Mais, si la révolution devait réussir et offrir un territoire officiel à ce peuple de vagabonds sans terre, il n'hésiterait pas à les trahir. En ce sens, Genet aurait pu devenir «l'ennemi intime de ses amis ${ }^{11}$ ». La seule fidélité qui lui importe étant celle qu'il éprouve pour lui-même et pour sa position d'homme en révolte, en marge, en « rejet de toutes les formes figées et institutionnelles », « contre les règles ${ }^{12} »$. En ce sens, Genet expose les enjeux d'un combat politique dont il ne partage pas les valeurs.

La question politique déserte l'espace dans le sens où les Palestiniens constitueraient pour Genet l'antithèse de toute politique comme l'étaient déjà les Black Panthers, antithèse ainsi de toute politique au sens étymologique du terme, c'est-à-dire sans cité, sans Polis ${ }^{13}$.

8. Patrice Bougon, «Politique et autobiographie», Le Magazine littéraire, $\mathrm{n}^{\circ}$ 313, septembre 1993, p. 69: "La visée du témoignage permet-elle de considérer Un captif amoureux comme un texte engagé? La position publique du narrateur est-elle vraiment aussi univoque qu'on a bien voulu le croire? [...] La position politique de Genet est moins propalestinienne qu'anarchiste. [...] Ce qui intéresse Genet chez les Black Panthers et les Feddayin, c'est qu'ils sont des vecteurs de déstabilisation du pouvoir et de l'État. »

9. Jean Genet, Un captif amoureux, op. cit., p. 37.

10. Ibid., p. 609.

11. Patrice Bougon, « Politique, ironie et religion dans Un captif amoureux », dans Bernard Alazet et Marc Dambre (dir.), Jean Genet : rituels de l'exhibition, Dijon, Éditions universitaires de Dijon, 2009, p. 82.

12. Eddie Breuil, « Genet enfant de chœur et correcteur de la Bible », article inédit. Nous remercions l'auteur de nous avoir communiqué cette étude.

13. Johan Faerber, «L'homme qui marchait dans la douleur, ou le désert de l'écriture dans Un captif amoureux de Jean Genet ", dans Bernard Alazet et Marc Dambre (dir.), op. cit., p. 94. 
Jérôme Neutres, reprenant une idée de Juan Goytisolo ${ }^{14}$, propose de remplacer, dans le cas de Genet, la notion de littérature engagée par celle de « littérature compromise ». Cette dernière se définirait comme « un investissement radical de l'écrivain dans une réalité politique », " fruit de la relation intime de l'écrivain avec la vie et l'écriture et non une adhésion extérieure à une quelconque cause politique ${ }^{15} \gg$.

La politique renvoie à ce qui concerne les citoyens et le gouvernement d'une cité, d'un État. Dans le cas des Palestiniens, l'État est à naître, il constitue une entité virtuelle dont les acteurs cherchent à faire émerger une réalité concrète. C'est sans nul doute cette création quasi mythique qui a pu séduire Genet et le conforter dans la théâtralité de l'affairisme politique en général. En somme, les Palestiniens font comme s'ils formaient un État. Ils jouent à être des gouvernants ou des soldats, de la même façon qu'ils jouent aux cartes avec des cartes imaginaires.

Un captif amoureux, le dernier récit de Jean Genet semble obéir au rituel de l'écrit engagé, en exhibant une prise de position politique en faveur des Palestiniens. Mais, comme souvent chez Genet, son discours déclaré a pour visée de nous masquer ce que son texte fait en contrebande ${ }^{16}$.

Cette remarque de Patrice Bougon souligne toute la complexité du discours genétien qui affirme tout en se contredisant, chaque parole effaçant la suivante et se superposant à elle. Genet, fantasque et malicieux, ne se plaît jamais autant que dans les situations de mystification où il peut mettre à mal son lecteur. Il lui demande de lire un texte, comme on le ferait d'un texte talmudique, en parallèle à d'autres textes qui le commentent et souvent s'y opposent. Cette ambiguitté initiale a été repérée par les premiers critiques de cette œuvre singulière qui articule de façon inédite une forme d'engagement et de témoignage historique et une forme autobiographique. Pour Jacques Henric, « [c'est] beaucoup plus et beaucoup mieux qu'un simple reportage, un pamphlet ou un livre de propagande. Genet traque en lui-même $s a$ vérité ${ }^{17} \gg$. Cette vérité ne peut qu'être plurielle pour Genet.

Alors qu'Un captif amoureux semble être une œuvre complètement tournée vers le monde extérieur, prolongeant la période d'engagement politique de Genet, l'écrivain parvient à opérer un retournement vers l'intime. « Il ne pose jamais en témoin capital qu'il fut » mais délivre « un joyeux testament ${ }^{18} »$, miroir de sa personne et de son écriture.

14. Juan Goytisolo, «Afghans et Palestiniens », Le Nouvel Observateur, mai 1981.

15. Jérôme Neutres, Genet sur les routes du Sud, Paris, Fayard, 2002, p. 246-247.

16. Patrice Bougon, "Politique, ironie et religion dans Un captif amoureux», op. cit., p. 81 .

17. Jacques Henric, Art Press, ${ }^{\circ}$ 106, septembre 1986, repris dans Agnès FontvieilleCordani et Dominique Carlat (dir.), op. cit., p. 149.

18. Jean-Pierre Léonardini, «L'exultation perpétuelle », L'Humanité, 18 juin 1986, repris dans Agnès Fontvieille-Cordani et Dominique Carlat (dir.), op. cit., p. 142. 


\section{Dans l'intimité d'un combat politique}

Grâce à l'étude lexicographique de Véronique Montémont ${ }^{19}$, l'histoire et les variations de sens de la notion d'intime apparaissent éclairées. C'est au double sens du mot intime que nous nous intéresserons dans le cas de Genet : union avec l'autre et intériorité du moi. Le premier sens est le sens le plus ancien; il associe « intime » à une relation d'amitié particulièrement profonde. Le second renvoie au sentiment d'une conscience de soi, d'une essence de soi qui demeure cachée. De ce jeu entre les deux sens naît le caractère hybride et indécidable du genre de l'œuvre. Un captif amoureux est à la fois un témoignage proche du genre des Mémoires ${ }^{20}$ et un récit autobiographique, ponctué de réflexions diverses sur l'histoire, les relations politiques, la religion, l'érotisme, la mort, l'art et l'écriture. Genet témoigne de son amitié pour les Palestiniens et en même temps y révèle une grande part de son moi le plus intime. Dans ses précédentes œuvres, il recourait à des masques et à des doubles pour parvenir à se dire. Dans $U n$ captif amoureux, Genet ne se cache plus, il parle bien en son nom, sans facéties. Il se distingue nettement de ceux qu'il observe et dont il relate les exploits.

Genet, témoin du combat palestinien, multiplie les allégations d'authenticité, de vérité historique. «Dès ici, je dois prévenir le lecteur que mes souvenirs sont exacts, dans les faits, les événements, les dates mais les conversations sont recomposées ${ }^{21} \gg$. Certes, il glisse des dates, rappelle des événements clés. Il se revendique même du reportage, terme utilisé de façon récurrente. Mais la vision d'un Genet journaliste sur le terrain n'est qu'une image parodique, un piège pour le lecteur ${ }^{22}$. «Avant de l'écrire je m'étais juré de dire toute la vérité dans ce livre [...] Selon le droit français, [le témoin] a juré de dire la vérité, non de la dire aux juges. [...] Le témoin est seul. Il parle ${ }^{23}$. » Or, à qui s'adresse Genet dans ce livre si ce n'est à des juges, à des ennemis qui méconnaissent la cause palestinienne?

Genet entre dans une intimité profonde avec les Palestiniens. Il témoigne de leur action politique, militaire, mais plus encore de leur

19. Véronique Montémont, «Dans la jungle de l'intime: enquête lexicographique et lexicométrique (1606-2008) », Itinéraires. Littératures, textes, cultures, $\mathrm{n}^{\circ}$ 2009-4, « Pour une histoire de l'intime et de ses variations », p. 15-38.

20. Sur cette question, se référer à l'analyse de Pierre-Marie Héron à partir du dossier de presse rassemblé dans le recueil Genet et son lecteur, «Un captif amoureux et le genre des Mémoires », Littérature, nº 159, septembre 2010, p. 53-63.

21. Jean Genet, Un captif amoureux, op. cit., p. 52.

22. Hédi Khélil, De l'extranéité à l'altérité : figures de l'écriture dans l'œuvre de Jean Genet, Louvain-la-Neuve, Academia-Bruylant, 2003, p. 69 : «le livre de souvenirs défait la réalité politique racontée, écrit la difficulté de la restituer, en fragments ou en intégralité, et dresse devant les facilités et les leurres du reportage ou de la littérature mémoriale, les paravents de l'écriture ".

23. Jean Genet, Un captif amoureux, op. cit., p. 610. 
quotidien, de leurs traditions. C'est bien à découvrir l'intimité des feddayin que Genet nous convie. Or, parler d'eux lui permettra de parler de lui-même en se dévoilant de façon intime, en révélant des pans secrets de sa vie et de sa personnalité. Néanmoins, si Genet suit une trame chronologique, il en dévie fréquemment. Les dates et autres références temporelles semblent parfois plus proches du leurre que de la vérité historique. Genet s'amuse ainsi à donner à son texte l'apparence de Mémoires, mais les preuves qu'il dissémine à travers les pages sont à prendre avec un recul critique. Comme dans Journal du voleur, l'auteur reste du côté de la fiction, même lorsqu'il se réfère à des écrits autobiographiques.

La rencontre avec ce peuple naît d'un projet politique et aboutit à la mise au jour d'une intimité individuelle ayant trouvé son reflet dans l'image d'un peuple rebelle et vagabond. Finalement, si Genet s'intéresse aux Palestiniens, c'est qu'il s'est reconnu à travers eux, c'est qu'il a reconnu des êtres auxquels il est apparenté. Son œuvre est alors un moyen de leur rendre hommage. Il en allait de même dans les premiers récits de l'écrivain : l'ensemble de ses romans célèbre les criminels illustres ou fictifs qu'ils mettent en scène, faisant figure de véritables tombeaux littéraires. Miracle de la rose est dédié à tous les camarades de la colonie agricole et pénitentiaire de Mettray où Genet séjourna durant son adolescence; Pompes funèbres est écrit à la mémoire de Jean Decarnin, militant communiste, amant de Genet durant la Seconde Guerre mondiale; Journal du voleur magnifie les exclus et marginaux rencontrés lors des voyages du narrateur dans toute l'Europe et plus particulièrement en Espagne.

Harcamone est mort, Bulkaen est mort. Si je sors, comme après la mort de Pilorge, j'irai fouiller les vieux journaux. Comme de Pilorge, il ne me restera plus entre les mains qu'un article très bref, sur un mauvais papier, une sorte de cendre grise qui m'apprendra qu'il fut exécuté à l'aube. Ces papiers sont leur tombeau. Mais je transmettrai très loin dans le temps leur nom. [...] Et leur nom troublera comme la lumière nous trouble qui arrive d'une étoile morte il y a mille ans ${ }^{24}$.

Avec Un captif amoureux, la démarche se révèle assez différente. Certes, Genet rend hommage à des disparus, mais il évoque également bon nombre de vivants. L'urgence réside dans le fait qu'il est celui qui va disparaître, s'effacer. Le Captif sera sa dernière œuvre et Genet, luttant depuis plusieurs années contre la maladie, le sait.

Éclipser est un mot riche. Outre le soleil, plus visible si la lune l'éclipse, tout événement, homme, figure, éclipsés par d'autres ou d'autres choses reviennent régénérés, la disparition fût-elle brève a fait son œuvre de nettoyage, de polissage ${ }^{25}$.

24. Jean Genet, Miracle de la rose [1946], Paris, Gallimard, coll. « Folio », 2000, p. 376.

25. Jean Genet, Un captif amoureux, op. cit., p. 535. 


\section{Lorsque le politique s'inscrit au cœur de l'intime}

L'ouverture du livre peut déconcerter le lecteur qui s'attend aux paroles liminaires d'un mémorialiste lui présentant son projet d'écriture. Genet ne pose pas de pacte de lecture : il ouvre - et conclut - sa dernière œuvre par une réflexion sur l'empreinte, sur le rapport entre la page et les signes, la lisibilité et la transparence. Réflexion qui transcende le simple témoignage circonstancié. Cependant, il fait référence à sa vision de la révolution palestinienne. Mais, c'est pour avouer aussitôt qu'elle lui avait « échappé ». Il imbrique ensuite la réalité des territoires occupés à la vie quotidienne. L'enjeu politique semble absent, l'humain et l'intime priment.

[Les] territoires occupés n'étaient que du drame vécu seconde par seconde par l'occupé et par l'occupant. Leur réalité était l'imbrication fertile en haine et en amour, dans les vies quotidiennes, semblable à la translucidité, silence haché par des mots et des phrases ${ }^{26}$.

Vient ensuite la première évocation des femmes palestiniennes dont Genet souligne le rire et la force, avant de dresser le portrait de l'une de ses " guides », Nabila Nashashibi. Le premier récit concernant le séjour de l'écrivain dans les camps est celui d'un thé où il sera « un homme, seul parmi un groupe de femmes arabes ${ }^{27} \gg$. Est-ce vraiment la vision de celui qui observe l'Orient « à l'envers ${ }^{28} »$ ? Ou bien s'agit-il d'un regard inédit, celui d'un témoin qui voit tout autre chose que les autres?

\footnotetext{
C'est ainsi que l'écriture de Genet effectue un déplacement politique de sa position : devant la grandeur de l'événement historique, elle se tient au singulier, à l'infime qui lui permet de détraquer, par des rapprochements inattendus, l'enchaînement logique et chronologique, ou bien plutôt d'une certaine idée de l'histoire. Aux grands événements historiques, comme la Libération, la guerre d'Algérie ou le conflit israélo-palestinien, il oppose des images simples qui mettent en valeur ce que l'on oublie de regarder lorsque l'on se concentre sur la grandeur de l'événement historique. [...] Il se consacre de la sorte au plus intime ${ }^{29}[\ldots]$.
}

Pour reprendre une expression proustienne, Genet alterne l'emploi du télescope et de la lorgnette ${ }^{30}$; il oscille entre une vision à grande échelle et les détails les plus infimes.

Relatant un épisode crucial de réconciliation entre Arafat et le roi Hussein de Jordanie, Genet se focalise sur la fête qui accompagne

26. Ibid., p. 12.

27. Ibid., p. 13.

28. Ibid.

29. Melina Balcázar Moreno, « La Mémoire vive », Le Magazine littéraire, n 503, « Jean Genet. Un centenaire tourmenté », décembre 2010, p. 63.

30. Jean Genet, Un captif amoureux, op. cit., p. 149 : «d'une fenêtre ou d'une loge, et comme avec une lorgnette de nacre, j'aurai regardé la révolte des Palestiniens ». 
l'événement et plus particulièrement sur la danse des membres des deux camps. Cette autre forme d'affrontement joue en faveur des Bédouins, lourdement armés, "presque menaçants " face à des Palestiniens maladroits. Cette lecture d'une activité de divertissement, hors du champ de bataille, met au jour le rapport de force entre les deux partis. Mais Genet va plus loin en lisant dans cette danse la révolte future des Bédouins contre leur propre roi, contre l'Occident, et la proximité des deux peuples, qui pourraient être frères.

Les Bédouins dansaient avec autour d'eux le désert et la nuit des temps. Et je me demande encore si la danse des Bédouins bardés de poudre et de balles, allant de vigueur en vigueur, de rigueur en rigueur, un jour n'aurait pas le pouvoir de faire craquer ce qu'elle semblait défendre : la monarchie hachémite; au-delà d'elle l'Amérique, aller à la conquête du ciel, y rencontrer les feddayin, parlant leur langue ${ }^{31}$.

Cet exemple démontre également l'attention portée par Genet au langage des corps. Cette dimension de l'intime intervient dans son œuvre à travers de multiples références aux diverses parties corporelles, y compris à celles qui sont considérées comme les plus « intimes » : parties génitales, fesses et anus. Les humeurs, sang, sueur, urine, sperme, comme les odeurs, trouvent place dans ses écrits où la vérité des êtres passe par celle des corps. Si la provocation pointe, elle n'est pas centrale. Le corps, pour Genet, révèle l'homme et plus particulièrement dans Un captif amoureux, il révèle une posture politique. Ainsi, les feddayin, comme les Black Panthers auparavant, signalent par leur apparence une érotique guerrière qui influence grandement la réception de leur image. "La politique de ces groupes se lit d'abord dans leur geste, selon un code de signes physiques et sexuels ${ }^{32}$. » Ce sont d'ailleurs ces images qui se propageront dans le temps et survivront aux combattants eux-mêmes : " des images fabuleuses, agissantes à long, à très long terme, après la mort ${ }^{33}$ ». L'adjectif « agissantes » est central : Genet affirme bel et bien la primauté du pouvoir de l'image et par conséquent des mots sur les actes eux-mêmes. Dans toute son œuvre, Genet aura cherché à montrer cette articulation entre le corps, son image et son pouvoir, des Figures du Balcon en passant par le Pape dans Elle; déjà dans ses romans, c'est par le corps désirant qu'il démontre le rôle de Hitler, des miliciens...

Tout fait sens et emblème : la mèche de Hitler, le cigare de Churchill, le coussin rose dans lequel Marx écrit Le Capital « contre » les commodes pansues de son appartement bourgeois, ou les colonnes aboulées d'Asie mineure, contraires au luxe puisqu'elles sont l'histoire même. Pour Genet, les conflits de tendance au sein de l'OLP comptent moins que le geste

31. Ibid., p. 115.

32. Jérôme Neutres, op. cit., p. 108.

33. Jean Genet, Un captif amoureux, op. cit., p. 429. 
élégant d'un lanceur de dés, ou les nuances d'accent et leurs trajets à travers 1'Islam ou le Paris des $\operatorname{argots}^{34}$.

Comme le souligne Bertrand Poirot-Delpech, Genet aborde la politique par des notations de détail mais qui sont autant $d^{\prime}$ ' « emblèmes », de symboles trahissant la vérité des hommes politiques, comme des engagements. Sa vision peut surprendre mais elle rivalise avec celle des analystes qu'elle prend à contre-pied.

\section{Genet dans sa vérité}

Le Captif offre à Genet la possibilité de remonter le cours de sa mémoire et de relier passé proche et passé lointain en un même réseau. L'intime prend une nouvelle dimension. Il quitte l'érotisme ${ }^{35}$ qui baignait les premiers récits pour se centrer sur le noyau le plus secret, le plus préservé de l'écrivain, celui de l'enfance. Cet intime qui se dit dans un livre à publier et qui sera donc exposé, partagé, reflète parfaitement l'évolution du mot lui-même :

[On] peut repérer un premier déplacement latéral, qui recentre l'intimité du relationnel vers l'individu, puis un mouvement d'expansion qui accroît le territoire de l'intime et le fixe comme un périmètre protégé, mais partageable ${ }^{36}$.

Si les feddayin lui rappellent les voyous ${ }^{37}$, tant aimés dans sa jeunesse, c'est en effet surtout son enfance qui affleure dans le récit, surgissant de façon inattendue et parfois cocasse : à travers l'église d'Alligny, la salle de classe, les sensations liées à la nature... On a affaire à de véritables phénomènes de réminiscences ${ }^{38}$. «[Chaque] souvenir est vrai. Une bouffée de fraîcheur redonne une vie fugitive à l'instant passé, passé définitivement. $[\ldots]$ Mais un livre de souvenirs est aussi peu vrai qu'un roman ${ }^{39}$. »

Ils rejetèrent mes couvertures et me sortirent de la tente. Si je les suivais en montant un chemin entre les noisetiers pendant deux kilomètres, je verrais la ferme et la fermière. Au sud du Jourdain, les collines près d'Ajloun sont

34. Bertrand Poirot-Delpech, «Le théâtre des opérations », Le Monde des livres, 6 juin 1986, repris dans Agnès Fontvieille-Cordani et Dominique Carlat (dir.), op. cit., p. 129.

35. Jean Genet, Un captif amoureux, op. cit., p. 294-295: «Les deux premiers feddayin étaient si beaux que je m'étonnai moi-même de n'avoir aucun désir pour eux, et plus je connus les soldats palestiniens en armes, ornés par elles, costumés léopard, bérets rouges sur l'œil, tels enfin que chacun paraissait non seulement la transfiguration de mes fantasmes mais leur matérialisation m'attendant là, devant moi, et, “comme s'ils" m'étaient offerts. » 36. Véronique Montémont, op. cit., p. 37.

37. Jean Genet, Un captif amoureux, op. cit., p. 287, p. 333.

38. Myriam Bendhif-Syllas, Genet, Proust : Chemins croisés, Paris, L'Harmattan, 2010, p. 233-245 et p. 362-364.

39. Jean Genet, Un captif amoureux, op. cit., p. 490-491. 
pareilles aux collines morvandelles. Quelquefois un pied de digitale, un chèvrefeuille, mais dans les prés moins de tracteurs, et pas une vache ${ }^{40}$.

Genet relate ainsi de nombreux détails, en apparence anodins, mais qui témoignent à la fois de sa mémoire la plus intime et du lien étroit qu'il entretient avec les Palestiniens, ses amis. "[L'on] sait que l'intime est d'abord fait d'infime, de petits riens qui constituent le sujet qui s'écrit ${ }^{41}$. » En ce sens, Genet s'écrit tout en écrivant les Palestiniens, il ne peut même s'écrire qu'en écrivant sur eux.

Genet, vieil homme au sein de la jeunesse palestinienne, se peint paradoxalement comme un enfant, rose et joufflu, fils d'une femme palestinienne qui n'est pas sa mère et d'un combattant afro-américain plus jeune que lui. C'est bien sa propre jeunesse qu'il retrouve et recompose au sein de ces peuples. Il ne s'agit pas d'un simple bain de jouvence ou d'un retour en enfance mais au contraire de la possibilité pour l'orphelin de se constituer le fils d'une famille d'élection, librement choisie et rejointe. Avec humour, il revient d'ailleurs à plusieurs reprises sur la nativité christique qu'il se plaît à confondre avec la sienne. Enfin et surtout, la figure de la Pièta ${ }^{42}$ le définit comme un fils dans les bras de sa mère. Cette figure centrale renvoie au couple auquel Genet se réfère dans tout le Captif et dont la recomposition fait l'objet d'une véritable quête de par le monde ${ }^{43}$. C'est sans nul doute la rencontre d'Hamza et de sa mère qui renvoie Genet au secret le plus profondément ancré en lui, celui du rapport mère-fils. Rapport inversé dans son cas puisqu'il demeure le fils vivant d'une mère " morte » depuis toujours, depuis son abandon à quelques mois. Dans la maison même de ce duo de combattants, Genet recevra les soins habituellement apportés au fils par la mère. Intimité de la demeure et intimité de la chambre où il est laissé seul. Intimité du geste et du don d'un repas et d'une boisson. L'intériorité est bel et bien représentée par le lieu même, mais aussi par la référence au corps et à ses excrétions.

Il était près de onze heures. Je dis adieu à la mère et à la sœur. Elles préparaient l'une pour son fils, l'autre pour son mari, la nourriture du soir et de la nuit qui venait. Et ceci faisait partie de mes souvenirs de 1970 je dois l'écrire : ce fut dans les cabinets de cette petite maison palestinienne que j'appris à me passer de papier mais à utiliser proprement la bouteille d'eau. Puisque j'avais bu et mangé dans la maison, mon intimité avec elle fut totale ${ }^{44}$.

40. Ibid., p. 117.

41. Françoise Simonet-Tenant, « À la recherche des prémices d'une culture de l'intime », Itinéraires. Littérature, textes, cultures, n 2009-4, « Pour une histoire de l'intime et de ses variations », p. 46.

42. Jean Genet, Un captif amoureux, op. cit., p. 289-290.

43. Ibid., p. 284 : «Je parle ici du voyage que j'entreprendrai en juillet 1984. Essayer de retrouver la mère. Très discrètement. »

44. Jean Genet, Un captif amoureux, op. cit., p. 285-286. 
L'analyse de ce rapport entre Hamza et sa mère, ainsi que la compréhension de son importance pour lui, au sein du combat palestinien, sont troublantes.

Cela aurait peu d'importance mais le mystère est grand ici : le sceau de la Révolution palestinienne ne me fut jamais un héros palestinien, une victoire (celle de Karameh par exemple) mais l'apparition presque incongrue de ce couple : Hamza et sa mère [...]. Ce groupe, tant de fois répété, profondément chrétien, symbole de la douleur inconsolable d'une mère dont le fils était Dieu, comment pouvait-il m'apparaître, et si vite, avec la vitesse d'un coup de foudre, le symbole de la résistance palestinienne, ce qui serait assez explicable, mais au contraire « que cette révolte eut lieu afin que me hantât ce couple $»^{45}$ ?

Genet inverse la logique attendue : ce couple ne serait pas que le symbole de la révolution mais l'aboutissement de sa quête, la raison d'être de sa présence parmi les Palestiniens. Comme si toute la révolte s'était organisée pour lui seul. Cette impression sans nul doute excessive souligne cependant de façon pertinente à quel point l'écrivain a pu se reconnaître au sein de ce peuple, dans son esprit de révolte et jusqu'à sa conception des relations mère-fils. Le combat des Palestiniens est un combat à la mesure de Genet, il y aura retrouvé tout ce qui compte pour lui. «Autant avouer qu'avec eux je restais, et je ne sais pas comment, de quelle autre façon, le dire, dans mon propre souvenir ${ }^{46}$. »

Se joindre aux Palestiniens en 1970 était véritablement un acte politique. «Mais rester, c'était au-delà de ça, c'était déjà comme un acte d'amour ${ }^{47}$ ", souligne Leilla Shahid. On pourrait ajouter qu'écrire est bien au-delà. C'est au cœur de l'intime que Genet entend trouver sa vision de la Palestine. À cela, il n'apporte nulle justification, nulle explication satisfaisante, il se contente de délivrer son récit au lecteur. De la fréquentation des grands, il ne retient qu'un détail, qu'une anecdote. Des combattants, il ne relève que la beauté et le chant et non les faits d'armes.

La gloire des héros doit peu à l'immensité des conquêtes, tout à la réussite des hommages; L'Iliade plus que la guerre d'Agamemnon [...]. Demeurent seuls, les témoignages plus ou moins exacts mais toujours excitants, accordés aux siècles à venir par les conquérants ${ }^{48}$.

45. Ibid., p. 292-293.

46. Ibid., p. 347.

47. Leïla Shahid, citée par Mathieu Lindon, «Un livre qu'Arafat lui a demandé d'écrire », Libération, 26 mai 1986, repris dans Agnès Fontvieille-Cordani et Dominique Carlat (dir.), op. cit., p. 123.

48. Jean Genet, Un captif amoureux, op. cit., p. 14-15. 
En marge de l'histoire, se dessine une lecture qui lie l'intime et le politique, une création où réel et fiction ${ }^{49}$ se mêlent, à la fois ardente défense et position critique d'un homme qui reste en dehors et qui déploie le lexique de l'amour et de la captivité pour rendre compte de son expérience dans un « mémoire-miroir ${ }^{50} »$ aux reflets changeants.

Myriam Bendhif-Syllas

49. Jérôme Neutres, op. cit., p. 17 : « [Un captif amoureux serait un] retour à la fiction, comme si la politique conduisait à une impasse lorsqu'elle est encadrée par l'idéologie, comme si la vérité passait nécessairement par la fiction...».

50. Jean Genet, Un captif amoureux, op. cit., p. 542. 